\title{
Adipose depot-specific effects of 16 weeks of pioglitazone on in vivo adipogenesis in women with obesity: a randomised controlled trial
}

\author{
Ursula White $^{1}$ (D) Mark D. Fitch $^{2}$ (D) $\cdot$ Robbie A. Beyl ${ }^{3}$ (D) Marc K. Hellerstein ${ }^{2} \cdot$ Eric Ravussin $^{4}$ (D)
}

Received: 18 July 2020 / Accepted: 14 August 2020 / Published online: 1 October 2020

(C) Springer-Verlag GmbH Germany, part of Springer Nature 2020

\begin{abstract}
Aims/hypothesis In vitro and rodent studies suggest that pioglitazone, a thiazolidinedione, can promote adipogenesis in adipose tissue (AT); however, there is a lack of in vivo studies in humans to support these findings. The objectives of this randomised, placebo-controlled, parallel-arm trial were to test if pioglitazone stimulates in vivo adipogenesis in the subcutaneous adipose tissue depots and if these measures were related to metabolic health outcomes in women with obesity.

Methods Forty-one healthy women with obesity (20 black; 21 white; $29 \pm 6$ years; BMI $32.0 \pm 1.7 \mathrm{~kg} / \mathrm{m}^{2} ; 44.0 \pm 3.6 \%$ body fat) were randomised to consume $30 \mathrm{mg}$ /day of pioglitazone $(n=21)$ or placebo $(n=20)$ for 16 weeks. SAS v9.4 was used to generate the block randomisation code sequence (stored in password-protected files) with a 1:1 allocation ratio. The participants and study staff involved in assessing and analysing data outcomes were blinded to the group assignments. The trial was conducted at Pennington Biomedical Research Center and ended in 2016. At baseline and post-intervention, subcutaneous abdominal (scABD) and femoral (scFEM) AT biopsies were collected, and in vivo cellular kinetics (primary endpoint of the trial) were assessed by an 8 week labelling protocol of deuterium $\left({ }^{2} \mathrm{H}\right)$ into the DNA of adipose cells. Body composition was measured by dual-energy x-ray absorptiometry (DXA), scABD and visceral AT (VAT) by MRI, ectopic fat by ${ }^{1} \mathrm{H}-\mathrm{MRS}$, and insulin sensitivity by an OGTT.

Results After the 16 week intervention, there was a significant decrease in visceral fat (VAT:total abdominal AT [as a \%]; $p=0.002$ ) and an increase in the Matsuda index (i.e. improved insulin sensitivity; $p=0.04$ ) in the pioglitazone group relative to the placebo group. A significant increase in the formation of new adipocytes was observed in the $\operatorname{scFEM}(\Delta=3.3 \pm 1.6 \% ; p=0.04)$ but not the scABD $\operatorname{depot}(\Delta=2.0 \pm 2.1 \% ; p=0.32)$ in the pioglitazone group relative to the placebo group. No serious adverse events were reported. Conclusions/interpretation Pioglitazone may elicit distinct differences in in vivo adipogenesis in subcutaneous adipose depots in women with obesity, with increased rates in the protective scFEM.

Trial registration ClinicalTrials.govNCT01748994

Funding This study was funded by R01DK090607, P30DK072476, and R03DK112006 from the National Institute of Diabetes and Digestive and Kidney Diseases of the National Institutes of Health. U54 GM104940 from the National Institute of General Medical Sciences of the National Institutes of Health. The Robert C. and Veronica Atkins Foundation.
\end{abstract}

Keywords Abdominal · Adipocyte kinetics $\cdot$ Adipocyte turnover $\cdot$ Adipogenesis $\cdot$ Adipose tissue $\cdot$ Cellular kinetics $\cdot$ Femoral $\cdot$ Metabolic health $\cdot$ Obesity $\cdot$ Pioglitazone

Electronic supplementary material The online version of this article (https://doi.org/10.1007/s00125-020-05281-7) contains peer-reviewed but unedited supplementary material, which is available to authorised users.

Ursula White

ursula.white@pbrc.edu

Extended author information available on the last page of the article

\begin{tabular}{ll}
\multicolumn{2}{l}{ Abbreviations } \\
AT & Adipose tissue \\
DXA & Dual-energy x-ray absorptiometry \\
EMCL & Extramyocellular lipid \\
IHL & Intrahepatic lipid \\
IMCL & Intramyocellular lipid \\
PBRC & Pennington Biomedical Research Center \\
scABD & Subcutaneous abdominal \\
scFEM & Subcutaneous femoral \\
TAT & Total adipose tissue \\
VAT & Visceral adipose tissue
\end{tabular}




\section{Research in context}

\section{What is already known about this subject?}

- Studies suggest that pioglitazone, a thiazolidinedione, can promote adipogenesis in adipose tissue

- There is a lack of in vivo studies in humans to support these findings

\section{What is the key question?}

- Does pioglitazone stimulate in vivo adipogenesis in different subcutaneous adipose tissue depots in humans, and are these measures related to metabolic health outcomes?

\section{What are the new findings?}

- This study is the first to assess in vivo adipogenesis in metabolically distinct human adipose tissue depots in response to pioglitazone via the deuterium-labelling metabolic protocol

- Pioglitazone stimulates a significant increase in in vivo adipogenesis in the subcutaneous femoral depot but not the subcutaneous abdominal depot in women with obesity

\section{How might this impact on clinical practice in the foreseeable future?}

- These findings validate this in vivo metabolic protocol as a cutting-edge approach to assess adipose cell turnover in response to interventions in humans and provide new knowledge on the in vivo mechanistic actions of a current pharmacological treatment for type 2 diabetes, pioglitazone

\section{Introduction}

White adipose tissue (AT) expansion is characterised by adipocytes accumulating lipid and increasing in size (hypertrophy) and/or increasing in number via the differentiation of pre-adipocytes (hyperplasia or adipogenesis). Evidence suggests that the capacity of subcutaneous AT to expand can influence cardiometabolic health, as impaired adipogenesis may result in increased ectopic deposition, contributing to obesity-associated diseases such as insulin resistance and type 2 diabetes [1].

Pioglitazone, a member of the thiazolidinedione family and a potent activator of the transcription factor peroxisome proliferator-activated receptor (PPAR)- $\gamma$, has been shown to substantially enhance insulin sensitivity in vivo [2] and is currently used as an effective therapeutic agent for type 2 diabetes [3-5]. While the underlying biological mechanisms are unclear, pioglitazone is thought to improve cardiometabolic health in humans by the redistribution of AT [6], via a reduction in visceral AT (VAT) $[7,8]$ and an increase in lower-body fat [9], and by promoting AT expansion (i.e. adipogenesis) in subcutaneous depots. Pioglitazone has been shown to induce adipocyte differentiation and lipid accumulation in cultured mouse cell lines in vitro [10] and to enhance adipogenesis by increasing the number of small adipocytes in the fat pads of obese rodents [11]. In one clinical study, pioglitazone was reported to increase the proportion of small adipocytes in individuals with type 2 diabetes [12], while another study in nondiabetic individuals who were either overweight or obese showed a similar effect of pioglitazone, with an increased number of small adipocytes associated with improved insulin sensitivity [13].
Collectively, these data suggest that pioglitazone may exert its beneficial effects by stimulating the formation of new adipocytes in subcutaneous AT. However, there is a scarcity of in vivo data in humans to corroborate these findings. While in vitro approaches have provided insight into the mechanisms underlying adipogenesis, these studies do not provide a comprehensive in vivo assessment within the natural microenvironment of the adipose tissue. In addition, no studies have investigated the effects of pioglitazone on in vivo adipogenesis in distinct AT depots. Preferential accumulation of abdominal AT, mainly VAT but also subcutaneous abdominal (scABD) AT, is associated with obesity-related diseases [14, 15]. Conversely, lower-body fat depots, including gluteal and femoral (scFEM), are associated with lower health risk and are metabolically protective [16-18], as evidenced by data from numerous clinical and epidemiological studies [19-27]. These data suggest that inherent differences in physiology and characteristics are likely to exist among adipose depots [28].

We report here the primary endpoints of the Apple \& Pear study (ClinicalTrials.gov NCT01748994), as the baseline data have been previously reported [29-31]. The primary outcomes of the study were to compare in vivo adipogenesis rates in two subcutaneous adipose depots in response to a 16 week pioglitazone intervention and to determine if these measures were related to improved metabolic outcomes. We assessed in vivo cellular kinetics in subcutaneous AT from the scABD and scFEM depots using an 8 week deuterium $\left({ }^{2} \mathrm{H}\right)$ labelling protocol via ${ }^{2} \mathrm{H}_{2} \mathrm{O}$ administration [32], with ${ }^{2} \mathrm{H}$ incorporated into the DNA of pre-adipocytes and adipocytes. Forty-nine overweight or obese women were assessed at baseline and after 16 weeks of either pioglitazone ( $30 \mathrm{mg} /$ day) or placebo. 


\section{Research design and methods}

\section{Participant characteristics}

Healthy women, who were either overweight or obese, were recruited according to the following inclusion criteria: 1840 years of age, BMI $27-38 \mathrm{~kg} / \mathrm{m}^{2}$, WHR either $>0.84$ or $<0.77$, fasting plasma glucose $(\leq 6.1 \mathrm{mmol} / \mathrm{l})$, blood pressure $(\leq 140 / 90 \mathrm{mmHg})$, absence of major organ disease, normal screening urinary and blood laboratory tests, weight stability for $\geq 3$ months $( \pm 3.2 \mathrm{~kg})$, and no self-reported significant changes in diet or physical activity in the previous month. Exclusion criteria consisted of a history of diagnosed diabetes; chronic use of medications with potential metabolic effects (glucocorticoids, adrenergic agents, thiazolidinediones); or use of medications or procedures that cause weight gain or weight loss. Individuals with HIV, hepatitis B or hepatitis C were excluded, as were pregnant or breastfeeding women. The institutional review board of Pennington Biomedical Research Center (PBRC) approved all procedures, and all study participants gave written, informed consent.

\section{Study design}

The Apple \& Pear trial ('Cellular dynamics of subcutaneous fat distribution in obese women'; ClinicalTrials.gov NCT01748994) was a randomised, double-blind, placebocontrolled, parallel-arm trial conducted at Pennington Biomedical Research Center (PBRC). To enhance recruitment and enrolment, the WHR limits $(>0.84$ or $<0.77)$ were removed after the trial commenced to accept the full continuum of WHR. After screening, eligible women completed an 8 week ${ }^{2} \mathrm{H}_{2} \mathrm{O}$-labelling protocol (Weeks $-8-0$ ). At Week 0 (baseline), the women were randomised (simple randomisation with a $1: 1$ allocation ratio) to consume $30 \mathrm{mg} /$ day of pioglitazone or placebo for 16 weeks (Weeks 0-16) and completed an additional ${ }^{2} \mathrm{H}_{2} \mathrm{O}$-labelling protocol during the last 8 weeks of the intervention (Weeks 8-16). Participants were encouraged to avoid major dietary and physical activity changes throughout the study.

Pioglitazone (30 mg), purchased from a pharmacy, was repackaged into capsules by the pharmacist at PBRC, and the placebo was packaged in similar capsules. The pioglitazone and placebo were distributed to participants during visits every 4 weeks at PBRC. To monitor compliance, participants were required to return unused pills for counting during these visits. All participants included in the analysis remained compliant through the duration of the study. Potential adverse events were identified by participant self-report and collection of blood to monitor the levels of liver enzymes, alanine aminotransferase (ALT) and aspartate aminotransferase (AST). The nature and frequency of adverse events were similar between the placebo and pioglitazone groups.
To avoid requiring a third adipose biopsy in participants, we conducted an additional study to measure the residual ${ }^{2} \mathrm{H}$ enrichment remaining in adipose tissue from the first labelling period (Week 8). Specifically, six women ( $29 \pm 6$ years of age; BMI $32.2 \pm 4.7 \mathrm{~kg} / \mathrm{m}^{2}$ ) with similar age and BMI to Apple \& Pear study participants were enrolled to complete an 8 week ${ }^{2} \mathrm{H}_{2} \mathrm{O}$-labelling protocol, and adipose biopsies were collected at Week 0 (the end of the ${ }^{2} \mathrm{H}_{2} \mathrm{O}$ administration period) and at Week 8 (i.e. without a second 8 week ${ }^{2} \mathrm{H}_{2} \mathrm{O}$-labelling period). The average (mean) ${ }^{2} \mathrm{H}$ decay rates in the adipose DNA from these women were used to estimate residual label at 8 weeks in the Apple \& Pear dataset. Additional information about the inclusion and exclusion criteria, trial dates, changes to the study design, randomisation, adverse events and CONSORT diagram (Electronic supplementary material [ESM] Fig. 1) can be found in the ESM.

\section{Study procedures and clinical measurements}

8 week ${ }^{2} \mathrm{H}_{2} \mathrm{O}$ labelling protocol (Weeks -8 to 0 and 8 to 16) After enrolment in the study, aliquots of $99.9 \%{ }^{2} \mathrm{H}_{2} \mathrm{O}$ (Sigma Aldrich, St. Louis, MO, USA) were administered in sterile containers. Participants were instructed to drink $35 \mathrm{ml}$ doses three times per day for Week 1 (priming period), and two $35 \mathrm{ml}$ doses per day for Weeks 2-8. This protocol maintains near-plateau body ${ }^{2} \mathrm{H}_{2} \mathrm{O}$ enrichments (1.5-2.5\%) during the 8 weeks [32]. Compliance with ${ }^{2} \mathrm{H}_{2} \mathrm{O}$ intake was monitored through weekly return of empty vials for counting and urine collections for measurement of deuterium enrichment in body water.

${ }^{2} \mathrm{H}$ enrichments in body water (urine) were measured by isotope ratio mass spectrometry [33]. Urine samples were cleaned using activated charcoal and filtered. Isotope enrichment was directly analysed with an H-Device attached to a Delta V Advantage Mass Spectrometer (Thermo Fisher Scientific Life Sciences, Waltham, MA, USA). ${ }^{2} \mathrm{H}_{2} \mathrm{O}$ enrichments were calculated by comparison with standard curves generated by mixing $100 \%{ }^{2} \mathrm{H}_{2} \mathrm{O}$ with natural abundance ${ }^{2} \mathrm{H}_{2} \mathrm{O}$ in known proportions.

Anthropometric characteristics (Weeks $-8,0$ and 16) Anthropometric characteristics, including height, weight, WHR, mean arterial pressure (1/3 [systolic BP - diastolic $\mathrm{BP}]+$ diastolic BP) were taken at the start of the study (Week -8), after the first 8 week ${ }^{2} \mathrm{H}$-labelling period (Week 0 ), and at the end of the 16 week study intervention (Week 16) following an overnight fast. Fasting plasma total triacylglyerol, HDL-cholesterol, LDL-cholesterol and total cholesterol were also measured using immunoassays with chemiluminescent detection (Siemens; Immulite 2000).

Body composition measures (Weeks 0 and 16) Dual-energy Xray absorptiometry (DXA) was performed after an overnight 
fast using the GE Lunar iDXA system to determine total percent body fat, from which fat mass and fat-free mass were calculated. Scans were analysed with the enCORE software, version 13.60.033. VAT and scABD AT volumes were defined and quantified with MRI using a $3.0 \mathrm{~T}$ scanner (GE, Discovery $750 \mathrm{w}$ ) by obtaining $\sim 581$ images from the dome of the liver to the pubic symphysis. Images were analysed by a single trained analyst. Estimates of VAT and scABD AT volumes were converted to mass using an assumed density of $0.92 \mathrm{~kg} / \mathrm{l}$. VAT:total abdominal AT (TAT) reflects the percentage of abdominal fat that is VAT and is calculated as $\mathrm{VAT} /(\mathrm{scABD} \mathrm{AT}+\mathrm{VAT})$.

Intramyocellular lipid (IMCL) and extramyocellular lipid (EMCL) in the soleus and anterior tibialis skeletal muscles, as well as intrahepatic lipid (IHL), were measured with proton magnetic resonance spectroscopy $\left({ }^{1} \mathrm{H}-\mathrm{MRS}\right)$ on a $3.0 \mathrm{~T}$ whole body imaging and spectroscopy system (GE, Discovery 750w) using a commercially available ${ }^{1} \mathrm{H}$ body coil. IMCL, EMCL and IHL content were determined with jMRUI (JavaBased Magnetic Resonance User Interface) [34] as previously described [35-37]. IMCL, EMCL and IHL peak areas are expressed relative to the peak area of a peanut oil phantom signal of known constant concentration, as previously described [38]. IMCL and EMCL data reflect the combination of soleus and anterior tibialis lipid.

OGTT (Weeks 0 and 16) Insulin sensitivity was estimated by the $2 \mathrm{~h}$ OGTT and calculated using the Matsuda index [39].

Adipose tissue biopsy collections (Weeks 0 and 16) AT biopsies were collected via needle lipoaspiration technique under sterile conditions and local anaesthesia [29]. Biopsy samples were taken from the scABD region, between one- and twothirds of the distance from the iliac spine to the umbilicus, and from the scFEM region, on the anterior aspect of the thigh, one- to two-thirds of the distance from the superior iliac spine to the patella. The tissue was immediately placed in sterile tubes for processing.

Isolation of adipocytes scABD and scFEM tissues were treated with a HEPES/type 1 collagenase solution for $1 \mathrm{~h}$ at $37^{\circ} \mathrm{C}$ to separate adipocytes and the stromal-vascular fraction [40]. As previously described [41], the adipocytes were purified by incubation with a cocktail of biotinylated antibodies against markers of endothelial cells (anti-human CD31; eBioscience, Thermo Fisher Scientific Life Sciences, Waltham, MA, USA; 1:200), haematopoietic cells (anti-human CD45; BioLegend, San Diego, CA, USA; 1:400), and mesenchymal stem cells (anti-human CD34; eBioscience, Thermo Fisher Scientific Life Sciences, Waltham, MA, USA 1:00) for $15 \mathrm{~min}$ at room temperature using an EasySep Cell Separation kit (EasySep, Stem Cell Technologies, USA). Cells attached to these antibodies were bound by magnetic nanoparticles (EasySep) and removed, while immuno-purified adipocytes were retained. Adipocytes were flash frozen in liquid $\mathrm{N}_{2}$ and stored at $-80^{\circ} \mathrm{C}$ until DNA extraction.

Collection of blood monocytes Blood was collected in FicollPaque (Cytiva, Marlborough, MA, USA) solution and the mononuclear fraction was removed after centrifugation. Monocytes were isolated as $\mathrm{CD} 14^{+}$cells by immunemagnetic beads (EasySep).

DNA preparation DNA was isolated from the adipocytes and blood monocytes using DNEasy microDNA extraction kits (Qiagen, Hilden, Germany). DNA was enzymatically hydrolysed to free deoxyribonucleosides [42] and hydrolysates were derivatised to pentafluorobenzylhydroxylamine (PFBHA) derivatives for GC-MS analysis.

\section{GC-MS analyses and calculation of fraction of new adipocytes} The PFBHA derivatives were analysed for incorporation of ${ }^{2} \mathrm{H}_{2} \mathrm{O}$ on an Agilent 6890/5973 GC-MS (Santa Clara, CA, USA) equipped with a $30 \mathrm{~m}$ DB-225 column using methane negative chemical ionisation and collecting ions in selected ion-monitoring mode at $\mathrm{m} / \mathrm{z} 435,436$ and 437 (representing $\mathrm{M}_{0}, \mathrm{M}_{1}$ and $\mathrm{M}_{2}$ mass isotopomers). Baseline (unenriched) DNA standards were measured concurrently to correct for abundance sensitivity.

The percentage enrichment of the $\mathrm{M}_{1}$ ion above natural abundance in the adipocytes was determined by subtracting the $\% \mathrm{M}_{1}$ in natural abundance standards from the $\% \mathrm{M}_{1}$ in the sample. The theoretical maximum $\mathrm{M}_{1}$ enrichment in adipose cells was calculated using mass isotopomer distribution analysis (MIDA) equations [43] based on the body ${ }^{2} \mathrm{H}_{2} \mathrm{O}$ exposure integrated over the 8 week period. The fraction of new cells (\%), as reported in the Results section, is calculated by the following:

Fraction of new cells $(\%)$

$$
=\frac{\mathrm{M}_{1} \text { enrichment in sample cells }}{\text { Theoretical maximum } \mathrm{M}_{1} \text { enrichment }} \times 100
$$

Blood monocytes were collected and analysed to represent a (near) completely turned-over cell population. This measurement, used as a reference marker of ${ }^{2} \mathrm{H}_{2} \mathrm{O}$ exposure in each individual, serves to confirm calculations using the theoretical maximum enrichment from the measured body $\mathrm{H}_{2} \mathrm{O}$ values [42].

\section{Statistical analysis}

Analyses were performed using SAS (version 9.4; SAS Institute, Cary, NC, USA) with a significance level of $\alpha=$ 0.05 . Due to budgetary constraints by the funder, the trial ended after $n=23$ (placebo group) and $n=26$ (pioglitazone 
group) participants completed the study in each group. Data were analysed using linear mixed-effects models, adjusted for treatment group and time as fixed effects. Differences in the fraction of new adipocytes (\%), or the formation of adipocytes, during the 8 -week ${ }^{2} \mathrm{H}$-labelling periods from the scABD and scFEM depots were tested using the least square means based on the linear mixed-effects model. Additional analyses were performed by including race as a fixed effect, but these analyses did not change the study outcomes. With the exception of Table 1 and unless otherwise stated, all results are reported as the least-squares means \pm SEM. Treatment effects, which represent the change induced in the pioglitazone group relative to the change in the placebo group, are denoted with the symbol ' $\Delta$ '.

\section{Results}

The analyses included 41 women who completed the study and had adipose tissue data available $(n=20$ in the placebo group; $n=21$ in the pioglitazone group) who were black ( $n=$ $20)$ or white $(n=21)$. They were $29 \pm 6$ years of age with a mean BMI of $32.0 \pm 1.7 \mathrm{~kg} / \mathrm{m}^{2}$ and $44.0 \pm 3.6 \%$ body fat. The clinical and metabolic characteristics of participants in the placebo and pioglitazone groups are included in Table 1.

Figure 1 demonstrates that the change (increase) in the fraction of new adipocytes in the pioglitazone group was significant in the scFEM $(\Delta=3.3 \pm 1.6 \% ; p=0.04)$ but not the scABD depot $(\Delta=2.0 \pm 2.1 \% ; p=0.32)$ compared with the placebo group. Table 2 provides measures of the formation of new adipocytes (i.e. in vivo adipogenesis) for each group. Additional analyses were performed to adjust for clinical covariates (i.e. body composition, ectopic fat and cardiometabolic risk factors) using Akaike's information criterion (AIC) for model selection. These analyses did not change the study outcomes and are not presented.

A linear mixed-effects model was used to determine the clinical and metabolic covariates associated with the change in the fraction of new adipocytes. Baseline scABD AT mass (Week 0) was positively associated with the change in the fraction of new adipocytes in the pioglitazone vs the placebo group in the scFEM depot (pioglitazone, $r=0.22$; placebo, $r=-0.34 ; p=0.03$ ) but not in the scABD depot (pioglitazone, $r=0.31$; placebo, $r=-0.07 ; p=0.15$ ). In addition, baseline VAT:TAT was negatively correlated with the change in the fraction of new adipocytes between the treatment groups in the scFEM depot (pioglitazone, $r=-0.63$; placebo, $r=-0.08$; $p=0.02$ ) but not the scABD depot (pioglitazone, $r=-0.21$; placebo, $r=-0.32 ; p=0.85$ ). The baseline characteristics for cardiovascular risk factors of participants in the placebo vs pioglitazone groups are shown in ESM Table 1. There were no associations between these covariates and changes in the fraction of new adipocytes (data not shown).

\section{Discussion}

Although previous studies have reported broad effects of thiazolidinediones on AT metabolism [44], this study is the first to assess in vivo cellular kinetics in human AT depots in response to the thiazolidinedione pioglitazone. Furthermore, no studies to date have examined in vivo cellular kinetics in metabolically distinct subcutaneous AT depots in response to a pioglitazone intervention. Our data provide evidence that pioglitazone stimulates a significant increase in in vivo adipogenesis in the scFEM depot but not the scABD depot in

Table 1 Baseline, treatment and changes in the clinical and metabolic characteristics of participants in the placebo and pioglitazone groups

\begin{tabular}{|c|c|c|c|c|c|c|c|c|}
\hline & PLB Week 0 & PLB Week 16 & $\begin{array}{l}p \text { value } \Delta \\
\text { Week } 0 \text { vs } 16\end{array}$ & PIO Week 0 & PIO Week 16 & $\begin{array}{l}p \text { value } \Delta \\
\text { Week } 0 \text { vs } 16\end{array}$ & $\Delta \mathrm{PLB}$ vs $\mathrm{PIO}$ & $\begin{array}{l}p \text { value } \Delta \\
\text { PLB vs PIO }\end{array}$ \\
\hline BMI $\left(\mathrm{kg} / \mathrm{m}^{2}\right)$ & $32.0 \pm 0.6$ & $32.6 \pm 0.7$ & 0.03 & $32.1 \pm 0.6$ & $32.7 \pm 0.7$ & 0.05 & $-0.1 \pm 0.3$ & 0.90 \\
\hline Body weight $(\mathrm{kg})$ & $86.8 \pm 2.0$ & $88.4 \pm 2.3$ & 0.04 & $86.1 \pm 1.9$ & $87.5 \pm 2.2$ & 0.05 & $-0.1 \pm 1.0$ & 0.92 \\
\hline Body fat $(\%)$ & $44.2 \pm 0.8$ & $44.8 \pm 0.8$ & 0.12 & $43.7 \pm 0.7$ & $44.1 \pm 0.8$ & 0.32 & $-0.2 \pm 0.5$ & 0.66 \\
\hline SAT (kg) & $7.9 \pm 0.3$ & $8.0 \pm 0.3$ & 0.54 & $7.8 \pm 0.3$ & $8.0 \pm 0.3$ & 0.15 & $0.1 \pm 0.2$ & 0.56 \\
\hline VAT (kg) & $7.1 \pm 0.9$ & $7.6 \pm 1.0$ & 0.06 & $6.5 \pm 0.9$ & $6.6 \pm 1.0$ & 0.53 & $-0.3 \pm 0.3$ & 0.36 \\
\hline VAT:TAT (\%) & $12.0 \pm 0.8$ & $12.5 \pm 0.8$ & 0.09 & $11.7 \pm 0.8$ & $10.8 \pm 0.8$ & 0.004 & $-1.32 \pm 0.3$ & 0.002 \\
\hline $\operatorname{IHL}(\%)$ & $2.4 \pm 1.1$ & $1.7 \pm 0.7$ & 0.57 & $4.2 \pm 1.0$ & $2.2 \pm 0.7$ & 0.11 & $-1.3 \pm 1.7$ & 0.47 \\
\hline IMCL (\%) & $0.5 \pm 0.1$ & $1.0 \pm 0.3$ & 0.06 & $0.5 \pm 0.1$ & $1.1 \pm 0.3$ & 0.03 & $0.1 \pm 0.3$ & 0.83 \\
\hline EMCL (\%) & $1.4 \pm 0.3$ & $3.0 \pm 0.9$ & 0.06 & $1.4 \pm 0.3$ & $3.1 \pm 0.9$ & 0.04 & $0.1 \pm 1.2$ & 0.96 \\
\hline Matsuda index & $4.7 \pm 0.5$ & $4.3 \pm 0.7$ & 0.35 & $4.2 \pm 0.5$ & $5.0 \pm 0.6$ & 0.05 & $1.1 \pm 0.5$ & 0.04 \\
\hline HOMA-IR & $2.2 \pm 0.4$ & $2.4 \pm 1.0$ & 0.84 & $2.7 \pm 0.4$ & $3.8 \pm 1.0$ & 0.22 & $0.9 \pm 1.3$ & 0.48 \\
\hline
\end{tabular}

Values presented as mean $\pm \mathrm{SD}$

PIO, pioglitazone group; PLB, placebo group; SAT, subcutaneous abdominal adipose tissue 


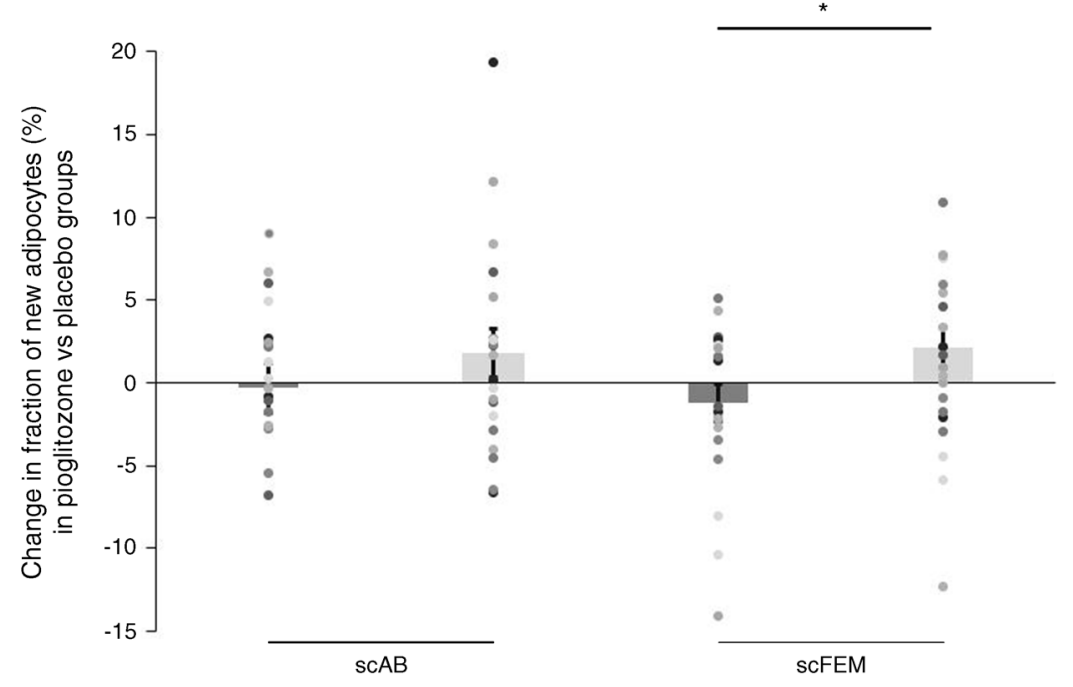

Fig. 1 The change in the fraction of new adipocytes was significant in the scFEM, but not the scABD depot in the pioglitazone group compared with the placebo group. The least square means comparing the change (post-intervention - baseline) in the fraction of new adipocytes in the pioglitazone vs the placebo group were derived from the linear mixedeffects model $(n=41)$. The change in the fraction of new adipocytes

between the pioglitazone and placebo groups in the scFEM depot is 3.3 $\pm 1.6 \%(p=0.04)$, while the change in the fraction of new adipocytes between the treatment groups in the scABD depot is $2.0 \pm 2.1 \%$ ( $p=$ 0.32 ). Values presented as means \pm SEM. Dark grey bars, placebo; light grey bars, pioglitazone

women with obesity, highlighting potential distinct mechanisms of action for pioglitazone on different subcutaneous adipose depots. As opposed to the abdominal AT depots, lower-body (e.g. scFEM) AT has been established as acting as a more protective energy storage depot [16-18]. Hence, our observations imply that pioglitazone may have a greater influence on the adipogenic rate in the metabolically protective scFEM depot $[16,17]$ than in the scABD depot in women with obesity.

In agreement with previous research [2, 13, 45], pioglitazone treatment induced a significant decrease in visceral fat (\% VAT:TAT; $p=0.002$ ) and an improvement in insulin sensitivity (Matsuda index; $p=0.04$ ) relative to the placebo (Table 1). Further analyses showed that baseline measures of abdominal adiposity were highly associated with the change in the fraction of new adipocytes in the scFEM depot. Interestingly, baseline subcutaneous AT mass was positively associated, while VAT:TAT was negatively associated, with the change in the fraction of new adipocytes between the treatment groups. These data suggest that higher visceral adiposity at baseline was correlated with a decreased capacity to make additional new adipocytes in response to

pioglitazone, while higher subcutaneous AT mass was associated with increased new adipocyte formation. Together, these data highlight subcutaneous adipose storage as an important predictor of adipocyte cellularity responses to pioglitazone.

Our present findings also highlight the varied perspectives regarding the role of adipogenesis in metabolic health. Data from our previous cross-sectional analyses challenge the AT expandability hypothesis, which proposes that higher adipogenesis (adipocyte formation) is associated with markers of impaired metabolic health [30]. We hypothesised an alternative manner of impaired adipose expansion (also suggested by other groups [46]), in which there could be an abundance of small adipocytes with a decreased capacity to sufficiently expand and store lipid, contributing to metabolic dysfunction in women with obesity. In contrast, pioglitazone treatment may stimulate the formation of adipocytes that are capable of adequate expansion and lipid storage in subcutaneous AT. Of note, pioglitazone has been previously reported to upregulate the expression of genes related to lipid synthesis and storage [47] and adipocyte differentiation [48] in the AT of humans.

Table 2 Proportion of new adipocytes in the scABD and scFEM depots of Apple \& Pear study participants

\begin{tabular}{lllllllll}
\hline & PLB Week 0 & PLB Week 16 & $\begin{array}{l}p \text { value } \Delta \\
\text { Week 0 vs 16 }\end{array}$ & PIO Week 0 & PIO Week 16 & $\begin{array}{l}p \text { value } \Delta \\
\text { Week 0 vs 16 }\end{array}$ & $\begin{array}{c}\Delta \text { PLB vs PIO } \\
p \text { value } \Delta \\
\text { PLB vs PIO }\end{array}$ \\
\hline scABD (\%) & $7.1 \pm 0.8$ & $6.8 \pm 1.1$ & 0.84 & $7.6 \pm 0.8$ & $9.3 \pm 1.1$ & 0.23 & $2.0 \pm 2.1$ \\
scFEM (\%) & $9.9 \pm 0.9$ & $8.7 \pm 0.8$ & 0.30 & $8.5 \pm 0.9$ & $10.6 \pm 0.9$ & 0.07 & $3.3 \pm 1.6$ & 0.32 \\
\hline
\end{tabular}

Values are presented as mean \pm SEM

PIO, pioglitazone group; PLB, placebo group 
Few studies have examined the direct effects of pioglitazone on subcutaneous AT in humans. One study reported that pioglitazone treatment increased the number of small adipocytes in the scABD AT of insulin-resistant men and women [13]. Contrary to this investigation, our RCT was conducted in a different study population (healthy, non-insulin-resistant women with obesity) and assessed both the scABD and scFEM fat depots. In addition, while the osmium fixation method was used to assess adipocyte cellularity [13], our study utilised a very different method $\left({ }^{2} \mathrm{H}\right.$-labelling) that provides quantitative measures of in vivo adipocyte formation. Nevertheless, we also observed improvements in insulin sensitivity (Matsuda index) and a decrease in visceral adiposity in the pioglitazone group (relative to the placebo group), which are consistent with findings from McLaughlin et al and other prior studies. To our knowledge, only one group has reported the effects of pioglitazone on the adipocyte morphology of the femoral depot [9]. The investigators showed a decrease $(p=0.07)$ in scFEM adipocyte size, despite an increase in leg fat mass, suggesting adipocyte proliferation (i.e. adipogenesis) rather than hypertrophy in the scFEM depot in response to pioglitazone in nondiabetic individuals with upper-body obesity [9]. Overall, findings from our study support the hypothesis that pioglitazone may exert its beneficial metabolic effects, in part, by stimulating the formation of new adipocytes in subcutaneous AT, especially in the scFEM AT depot.

An important study limitation is that kinetics in visceral AT were not assessed. Nevertheless, this study is strengthened by including two distinct depots. As the population was limited to overweight and obese women, our results cannot be extrapolated to cellular kinetics in men due to sex differences in adipose distribution and characteristics $[17,28]$. Another limitation was the use of data from a reference group of six women to correct for residual ${ }^{2} \mathrm{H}$-label from the first labelling period prior to the repeat metabolic labelling period. Though similar in age and BMI with the Apple \& Pear study participants, individual variability in ${ }^{2} \mathrm{H}$ decay rates in AT among individuals cannot be ruled out. Nevertheless, as the decay rates among the six women were consistent, it can be reasonably expected that these data provide reliable estimates for the correction analysis.

In conclusion, data from this randomised controlled trial (Apple \& Pear) depicts novel regional differences in in vivo adipose cellular kinetics in response to pioglitazone. We previously reported that scFEM AT may have a higher capacity for in vivo adipogenesis than scABD AT [29], providing additional evidence to implicate the thigh as a more efficient depot to expand and accommodate lipid $[16,17]$ in women with obesity. We now demonstrate that in vivo adipocyte formation rates in response to pioglitazone may also vary by depot location in this study population, with increased rates in the protective scFEM compared with the scABD depot. More studies are needed to further characterise the physiological effects of pioglitazone, as well as other pharmacological treatments for type 2 diabetes, on AT metabolism in order to better understand their influence on metabolic health.

Acknowledgements The authors thank C. Hebert (Human Physiology; PBRC) for laboratory and technical assistance. The authors thank the participants for their time and effort. The authors thank the PBRC Cores, including the Outpatient Unit (A. Eldredge), Pharmacy (C. Hazlett), Recruiting (A. Carville and G. Bella), Advertising (L. Giffin), Imaging (O. T. Carmichael and K. Murray), and Clinical Chemistry (S. Lee and S. Roussel).

Data availability All data generated or analysed during this study are included in this published article (and its ESM).

Funding This work was mostly supported by R01DK090607 (ER) and partially by a NORC Center Grant P30DK072476 (ER) from the National Institute of Diabetes and Digestive and Kidney Diseases of the National Institutes of Health. UW is supported in part by R03DK1 12006 from the National Institute of Diabetes and Digestive and Kidney Diseases of the National Institutes of Health. RAB is supported in part by U54 GM104940 from the National Institute of General Medical Sciences of the National Institutes of Health, which funds the Louisiana Clinical and Translational Science Center. MKH was partially supported by a gift from the Robert C. and Veronica Atkins Foundation. MKH has ownership interest in KineMed, Inc., which has no product in this area of research.

Authors' relationships and activities The authors declare that there are no relationships or activities that might bias, or be perceived to bias, their work.

Contribution statement UW assisted in the following: planning the study design; performing the experiments; acquisition of data; analysis, review, and interpretation of data; drafting and revising the manuscript critically for important intellectual content; and final approval of the manuscript version to be published. MDF provided the following: guidance for performing the in vivo adipogenesis method; acquisition of data; data analysis (DNA extraction and gas chromatography-mass spectrometry analysis) review and interpretation of data; revising the manuscript critically for important intellectual content; and final approval of the manuscript version to be published. RAB provided the following: conducted and described statistical methods; analysis and review of experimental data; revising the manuscript; and final approval of the manuscript version to be published. MKH provided the following: guidance for performing the in vivo adipogenesis method; data analysis (quality assurance of the GC-MS methods); review and interpretation of data; revising the manuscript critically for important intellectual content; and final approval of the manuscript version to be published. ER contributed the following: conceptualising and planning the study design; review and interpretation of data; guidance for developing and revising the manuscript critically for important intellectual content; and final approval of the manuscript version to be published. ER is the guarantor of this work.

\section{References}

1. Danforth E Jr (2000) Failure of adipocyte differentiation causes type II diabetes mellitus? Nat Genet 26(1):13. https://doi.org/10. $1038 / 79111$ 
2. Saltiel AR, Olefsky JM (1996) Thiazolidinediones in the treatment of insulin resistance and type II diabetes. Diabetes 45(12):16611669. https://doi.org/10.2337/diab.45.12.1661

3. Chiquette E, Ramirez G, Defronzo R (2004) A meta-analysis comparing the effect of thiazolidinediones on cardiovascular risk factors. Arch Intern Med 164(19):2097-2104. https://doi.org/10. 1001/archinte.164.19.2097

4. Inzucchi SE, Bergenstal RM, Buse JB et al (2015) Management of hyperglycaemia in type 2 diabetes, 2015: a patient-centred approach. Update to a position statement of the American Diabetes Association and the European Association for the Study of Diabetes. Diabetologia 58(3):429-442. https://doi.org/10.1007/ s00125-014-3460-0

5. Palmer SC, Mavridis D, Nicolucci A et al (2016) Comparison of clinical outcomes and adverse events associated with glucoselowering drugs in patients with type 2 diabetes: a meta-analysis. JAMA 316(3):313-324. https://doi.org/10.1001/jama.2016.9400

6. Pramyothin P, Karastergiou K (2016) What can we learn from interventions that change fat distribution? Curr Obes Rep 5(2): 271-281. https://doi.org/10.1007/s13679-016-0215-x

7. Miyazaki Y, Mahankali A, Matsuda M et al (2002) Effect of pioglitazone on abdominal fat distribution and insulin sensitivity in type 2 diabetic patients. J Clin Endocrinol Metab 87(6):27842791. https://doi.org/10.1210/jcem.87.6.8567

8. Basu A, Jensen MD, McCann F, Mukhopadhyay D, Joyner MJ, Rizza RA (2006) Effects of pioglitazone versus glipizide on body fat distribution, body water content, and hemodynamics in type 2 diabetes. Diabetes Care 29(3):510-514. https://doi.org/10.2337/ diacare.29.03.06.dc05-2004

9. Shadid S, Jensen MD (2003) Effects of pioglitazone versus diet and exercise on metabolic health and fat distribution in upper body obesity. Diabetes Care 26(11):3148-3152. https://doi.org/10. 2337/diacare.26.11.3148

10. Sandouk T, Reda D, Hofmann C (1993) Antidiabetic agent pioglitazone enhances adipocyte differentiation of 3T3-F442A cells. Am J Phys 264(6 Pt 1):C1600-C1608. https://doi.org/10.1152/ajpcell. 1993.264.6.C1600

11. Hallakou S, Doare L, Foufelle F et al (1997) Pioglitazone induces in vivo adipocyte differentiation in the obese Zucker fa/fa rat. Diabetes 46(9):1393-1399. https://doi.org/10.2337/diab.46.9.1393

12. Smith SR, Xie H, Baghian S, Needham A, McNeil M, Bogacka I, Bray GA (2006) Pioglitazone changes the distribution of adipocyte size in type 2 diabetes. Adipocyte 2:11-22

13. McLaughlin TM, Liu T, Yee G et al (2010) Pioglitazone increases the proportion of small cells in human abdominal subcutaneous adipose tissue. Obesity (Silver Spring) 18(5):926-931. https://doi. org/10.1038/oby.2009.380

14. Huang T, Qi Q, Zheng Y et al (2015) Genetic predisposition to central obesity and risk of type 2 diabetes: two independent cohort studies. Diabetes Care 38(7):1306-1311. https://doi.org/10.2337/ dc14-3084

15. Tchernof A, Despres JP (2013) Pathophysiology of human visceral obesity: an update. Physiol Rev 93(1):359-404. https://doi.org/10. 1152/physrev.00033.2011

16. Manolopoulos KN, Karpe F, Frayn KN (2010) Gluteofemoral body fat as a determinant of metabolic health. Int J Obes 34(6):949-959. https://doi.org/10.1038/ijo.2009.286

17. Karastergiou K, Fried SK (2012) Sex differences in human adipose tissues - the biology of pear shape. Biol Sex Differ 3(1):13. https:// doi.org/10.1186/2042-6410-3-13

18. Karpe F, Pinnick KE (2015) Biology of upper-body and lowerbody adipose tissue-link to whole-body phenotypes. Nat Rev Endocrinol 11(2):90-100. https://doi.org/10.1038/nrendo.2014. 185

19. Snijder MB, Visser M, Dekker JM et al (2005) Low subcutaneous thigh fat is a risk factor for unfavourable glucose and lipid levels, independently of high abdominal fat. The Health ABC Study. Diabetologia 48(2):301-308. https://doi.org/10.1007/s00125-004$1637-7$

20. Yim JE, Heshka S, Albu JB, Heymsfield S, Gallagher D (2008) Femoral-gluteal subcutaneous and intermuscular adipose tissues have independent and opposing relationships with CVD risk. J Appl Physiol (1985) 104(3):700-707. https://doi.org/10.1152/ japplphysiol.01035.2007

21. Snijder MB, Dekker JM, Visser M et al (2004) Trunk fat and leg fat have independent and opposite associations with fasting and postload glucose levels: the Hoorn study. Diabetes Care 27(2): 372-377. https://doi.org/10.2337/diacare.27.2.372

22. Snijder MB, Dekker JM, Visser M et al (2003) Larger thigh and hip circumferences are associated with better glucose tolerance: the Hoorn study. Obes Res 11(1):104-111. https://doi.org/10.1038/ oby. 2003.18

23. Seidell JC, Perusse L, Despres JP, Bouchard C (2001) Waist and hip circumferences have independent and opposite effects on cardiovascular disease risk factors: the Quebec Family Study. Am J Clin Nutr 74(3):315-321. https://doi.org/10.1093/ajcn/74.3.315

24. Snijder MB, Zimmet PZ, Visser M, Dekker JM, Seidell JC, Shaw JE (2004) Independent and opposite associations of waist and hip circumferences with diabetes, hypertension and dyslipidemia: the AusDiab Study. Int J Obes Relat Metab Disord 28(3):402-409. https://doi.org/10.1038/sj.ijo.0802567

25. Yusuf S, Hawken S, Ounpuu S et al (2005) Obesity and the risk of myocardial infarction in 27,000 participants from 52 countries: a case-control study. Lancet 366(9497):1640-1649. https://doi.org/ 10.1016/S0140-6736(05)67663-5

26. Bigaard J, Frederiksen K, Tjonneland A et al (2004) Waist and hip circumferences and all-cause mortality: usefulness of the waist-tohip ratio? Int J Obes Relat Metab Disord 28(6):741-747. https://doi. org/10.1038/sj.ijo.0802635

27. Canoy D, Boekholdt SM, Wareham N et al (2007) Body fat distribution and risk of coronary heart disease in men and women in the European Prospective Investigation Into Cancer and Nutrition in Norfolk cohort: a population-based prospective study. Circulation $116(25): 2933-2943$. https://doi.org/10.1161/ CIRCULATIONAHA.106.673756

28. Tchkonia T, Thomou T, Zhu Y et al (2013) Mechanisms and metabolic implications of regional differences among fat depots. Cell Metab 17(5):644-656. https://doi.org/10.1016/j.cmet.2013.03.008

29. White UA, Fitch MD, Beyl RA, Hellerstein MK, Ravussin E (2016) Differences in in vivo cellular kinetics in abdominal and femoral subcutaneous adipose tissue in women. Diabetes 65(6): 1642-1647. https://doi.org/10.2337/db15-1617

30. White UA, Fitch MD, Beyl RA, Hellerstein MK, Ravussin E (2017) Association of in vivo adipose tissue cellular kinetics with markers of metabolic health in humans. J Clin Endocrinol Metab 102(7):2171-2178. https://doi.org/10.1210/jc.2016-4000

31. White UA, Fitch MD, Beyl RA, Hellerstein MK, Ravussin E (2018) Racial differences in in vivo adipose lipid kinetics in humans. J Lipid Res. https://doi.org/10.1194/jlr.P082628

32. Neese RA, Misell LM, Turner S et al (2002) Measurement in vivo of proliferation rates of slow turnover cells by ${ }^{2} \mathrm{H}_{2} \mathrm{O}$ labeling of the deoxyribose moiety of DNA. Proc Natl Acad Sci U S A 99(24): $15345-15350$

33. Collins ML, Eng S, Hoh R, Hellerstein MK (2003) Measurement of mitochondrial DNA synthesis in vivo using a stable isotope-mass spectrometric technique. J Appl Physiol (1985) 94(6):2203-2211. https://doi.org/10.1152/japplphysiol.00691.2002

34. Naressi A, Couturier C, Devos JM et al (2001) Java-based graphical user interface for the MRUI quantitation package. MAGMA 12(23):141-152

35. Larson-Meyer DE, Heilbronn LK, Redman LM et al (2006) Effect of calorie restriction with or without exercise on insulin sensitivity, 
beta-cell function, fat cell size, and ectopic lipid in overweight subjects. Diabetes Care 29(6):1337-1344. https://doi.org/10.2337/ dc05-2565

36. Larson-Meyer DE, Smith SR, Heilbronn LK, Kelley DE, Ravussin E, Newcomer BR (2006) Muscle-associated triglyceride measured by computed tomography and magnetic resonance spectroscopy. Obesity (Silver Spring) 14(1):73-87. https://doi.org/10.1038/oby. 2006.10

37. Larson-Meyer DE, Newcomer BR, Heilbronn LK et al (2008) Effect of 6-month calorie restriction and exercise on serum and liver lipids and markers of liver function. Obesity (Silver Spring) 16(6): 1355-1362. https://doi.org/10.1038/oby.2008.201

38. Perseghin G, Scifo P, De Cobelli F et al (1999) Intramyocellular triglyceride content is a determinant of in vivo insulin resistance in humans: a $1 \mathrm{H}-13 \mathrm{C}$ nuclear magnetic resonance spectroscopy assessment in offspring of type 2 diabetic parents. Diabetes 48(8): 1600-1606. https://doi.org/10.2337/diabetes.48.8.1600

39. Matsuda M, DeFronzo RA (1999) Insulin sensitivity indices obtained from oral glucose tolerance testing: comparison with the euglycemic insulin clamp. Diabetes Care 22(9):1462-1470. https://doi.org/10.2337/diacare.22.9.1462

40. Tchoukalova YD, Koutsari C, Karpyak MV, Votruba SB, Wendland E, Jensen MD (2008) Subcutaneous adipocyte size and body fat distribution. Am J Clin Nutr 87(1):56-63. https:// doi.org/10.1093/ajen/87.1.56

41. Tchoukalova YD, Fitch M, Rogers PM et al (2012) In vivo adipogenesis in rats measured by cell kinetics in adipocytes and plasticadherent stroma-vascular cells in response to high-fat diet and thiazolidinedione. Diabetes 61(1):137-144. https://doi.org/10. 2337/db10-1768
42. Busch R, Neese RA, Awada M, Hayes GM, Hellerstein MK (2007) Measurement of cell proliferation by heavy water labeling. Nat Protoc 2(12):3045-3057. https://doi.org/10.1038/nprot.2007.420

43. Hellerstein MK, Neese RA (1992) Mass isotopomer distribution analysis: a technique for measuring biosynthesis and turnover of polymers. Am J Phys 263(5 Pt 1):E988-E1001

44. Boden G, Cheung P, Mozzoli M, Fried SK (2003) Effect of thiazolidinediones on glucose and fatty acid metabolism in patients with type 2 diabetes. Metabolism 52(6):753-759. https://doi.org/ 10.1016/s0026-0495(03)00055-6

45. Kodama N, Tahara N, Tahara A et al (2013) Effects of pioglitazone on visceral fat metabolic activity in impaired glucose tolerance or type 2 diabetes mellitus. J Clin Endocrinol Metab 98(11):44384445. https://doi.org/10.1210/jc.2013-2920

46. McLaughlin T, Sherman A, Tsao P et al (2007) Enhanced proportion of small adipose cells in insulin-resistant vs insulin-sensitive obese individuals implicates impaired adipogenesis. Diabetologia 50(8):1707-1715. https://doi.org/10.1007/s00125-007-0708-y

47. Bogacka I, Xie H, Bray GA, Smith SR (2004) The effect of pioglitazone on peroxisome proliferator-activated receptor-gamma target genes related to lipid storage in vivo. Diabetes Care 27(7):1660 1667. https://doi.org/10.2337/diacare.27.7.1660

48. Hammarstedt A, Sopasakis VR, Gogg S, Jansson PA, Smith U (2005) Improved insulin sensitivity and adipose tissue dysregulation after short-term treatment with pioglitazone in non-diabetic, insulin-resistant subjects. Diabetologia 48(1):96-104. https://doi. org/10.1007/s00125-004-1612-3

Publisher's note Springer Nature remains neutral with regard to jurisdictional claims in published maps and institutional affiliations.

\section{Affiliations}

\section{Ursula White $^{1}\left(\mathbb{D} \cdot\right.$ Mark D. Fitch $^{2}(\mathbb{D}) \cdot$ Robbie A. Beyl $^{3}\left(\mathbb{D} \cdot\right.$ Marc K. Hellerstein $^{2} \cdot$ Eric Ravussin $^{4}$ (D)}

1 Physiology of Human Adipose Tissue, Pennington Biomedical Research Center, Louisiana State University System, Baton Rouge, LA, USA

2 Nutritional Sciences and Toxicology, University of California at Berkeley, Berkeley, CA, USA
3 Biostatistics, Pennington Biomedical Research Center, Louisiana State University System, Baton Rouge, LA, USA

4 Human Translational Physiology, Pennington Biomedical Research Center, Louisiana State University System, Baton Rouge, LA, USA 